\title{
Nonautomatic emotion perception in a dual-task situation
}

\author{
DaVe Tomasik AND ERIC RUTHRUFF \\ University of New Mexico, Albuquerque, New Mexico \\ Philip A. Allen \\ University of Akron, Akron, Ohio \\ AND \\ Mei-Ching LiEN \\ Oregon State University, Corvallis, Oregon
}

\begin{abstract}
Are emotions perceived automatically? Two psychological refractory period experiments were conducted to ascertain whether emotion perception requires central attentional resources. Task 1 required an auditory discrimination (tone vs. noise), whereas Task 2 required a discrimination between happy and angry faces. The difficulty of Task 2 was manipulated by varying the degree of emotional expression. The stimulus onset asynchrony (SOA) between Task 1 and Task 2 was also varied. Experiment 1 revealed additive effects of SOA and Task 2 emotion-perception difficulty. Experiment 2 replicated the additive relationship with a stronger manipulation of emotion-perception difficulty. According to locus-of-slack logic, our participants did not process emotional expressions while central resources were devoted to Task 1. We conclude that emotion perception is not fully automatic.
\end{abstract}

When trying to perform two or more speeded tasks at nearly the same time, people usually have great difficulty. The robustness of this dual-task interference across a wide range of tasks led investigators to propose that central attentional resources can be allocated to only one task at a time, creating a processing bottleneck. Although the basic concept of a processing bottleneck has been supported, it remains unclear exactly which mental operations require central attentional resources. Early work focused on response selection, but it was later discovered that certain challenging perceptual stages (e.g., word identification; McCann, Remington, \& Van Selst, 2000) also require central resources. The present article addresses the relatively unexplored issue of whether the perception of emotional expressions requires central attentional resources. Although emotional processes seem effortless and beyond conscious control, we will present new data suggesting that they are not fully automatic in dual-task situations.

\section{Background: The Central Bottleneck Model of the PRP Effect}

Dual-task performance is typically investigated by giving participants two speeded tasks (Task 1 and Task 2) and measuring the response time (RT). In the widely used psychological refractory period (PRP) paradigm, the critical independent variable is the stimulus onset asynchrony (SOA) - the time between the onsets of the stimuli for
Task 1 and Task 2. Typically, Task 2 RT increases sharply as SOA decreases - a phenomenon known as the PRP effect.

Although there have been many accounts of the PRP effect, the dominant theory at present is the central bottleneck model (Pashler, 1984; but see also Meyer \& Kieras, 1997; Tombu \& Jolicœur, 2003). This model, shown in Figure 1, asserts that central stages (such as response selection) for Task 1 and Task 2 operate in series. At short SOAs, Task 1 accesses central attentional resources first, forcing Task 2 central stages to wait and creating a period of "cognitive slack" in the processing of Task 2 (represented by dots in Figure 1).

The existence of a processing bottleneck is well supported by a wide range of PRP experiments (see, e.g., McCann \& Johnston, 1992; Pashler, 1984; Pashler \& Johnston, 1989). It is less clear, however, which mental processes are subject to this bottleneck. The vague term central is merely a placeholder; only by empirically testing a wide range of processing stages can we accurately characterize the scope and nature of the bottleneck.

Fortunately, there is a well-established method - known as locus-of-slack logic-for determining which operations are subject to the processing bottleneck. The basic approach is to manipulate the duration of the target operation on Task 2 within the PRP paradigm. Figure 2 shows two possible outcomes. If the manipulated Task 2 stage

E. Ruthruff, ruthruff@unm.edu 
Long SOA
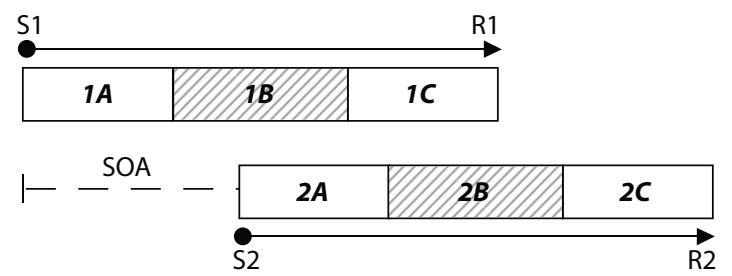

Short SOA

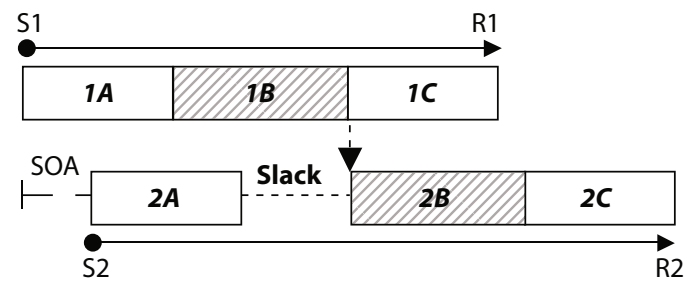

Figure 1. The central bottleneck model assumes that central stages (1B and 2B) cannot operate at the same time. Consequently, responses to Task 2 are slowed at short stimulus onset asynchronies (SOAs) relative to long SOAs. $1 \mathrm{~A}, 1 \mathrm{~B}$, and $1 \mathrm{C}$ are the perceptual, central, and response initiation/execution stages of Task 1, respectively. 2A, 2B, and $2 \mathrm{C}$ are the corresponding stages for Task 2. S1, Task 1 stimulus; S2, Task 2 stimulus; R1, Task 1 response; R2, Task 2 response. (indicated by the box with arrows) occurs prior to the bottleneck (i.e., is "automatic"), then the effects of this manipulation should be much smaller at short SOAs than at long SOAs, perhaps disappearing entirely. This pattern is called an underadditive interaction (see Figure 2A). Schweickert (1978) referred to this as a slack effect, because the additional Task 2 processing is absorbed into the cognitive slack created by the bottleneck at short SOAs. But, if the manipulated Task 2 stage occurs at or after the bottleneck (see Figure 2B), then the effects of the difficulty manipulation should be additive with the effects of SOA (because no absorption into slack is possible at any SOA). Thus, by determining how a particular Task 2 manipulation interacts with SOA, it is possible to discover whether the manipulated stage requires central attentional resources.

Early studies using locus-of-slack logic showed that response selection is subject to the bottleneck (see, e.g., McCann \& Johnston, 1992; see Lien \& Proctor, 2002, for a review). More recent studies have widened the scope of the central bottleneck to include word identification (e.g., Lien, Ruthruff, Cornett, Goodin, \& Allen, 2008; McCann et al., 2000), memory encoding (Jolicœur, 1998), mental rotation (Ruthruff, Miller, \& Lachmann, 1995), memory retrieval

A
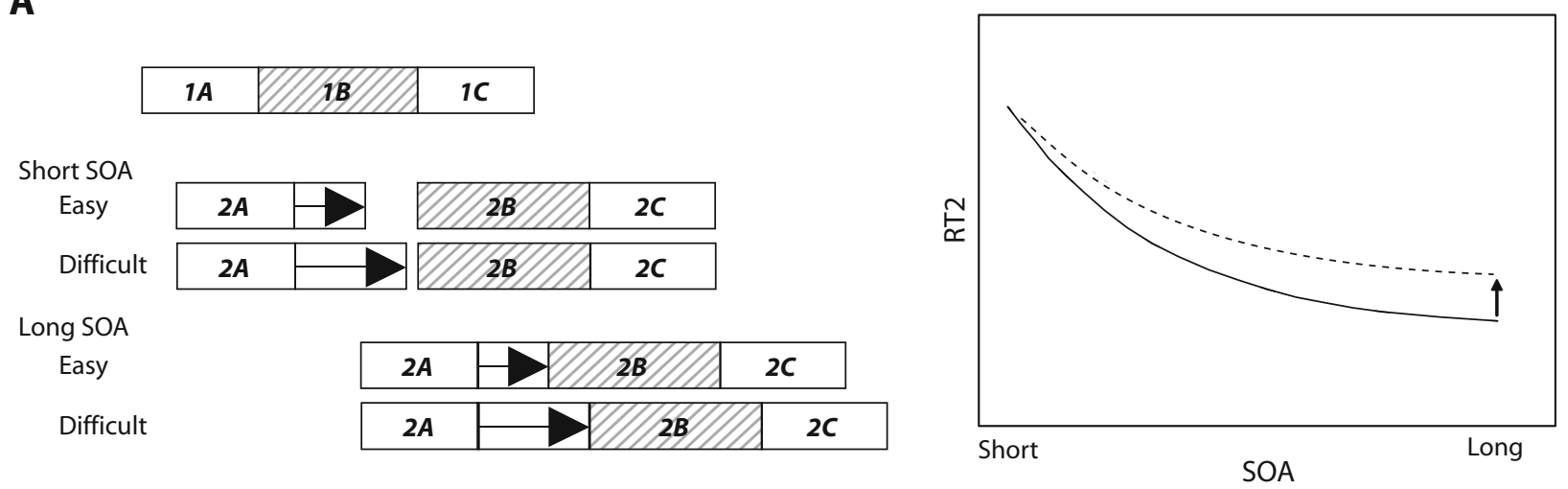

B
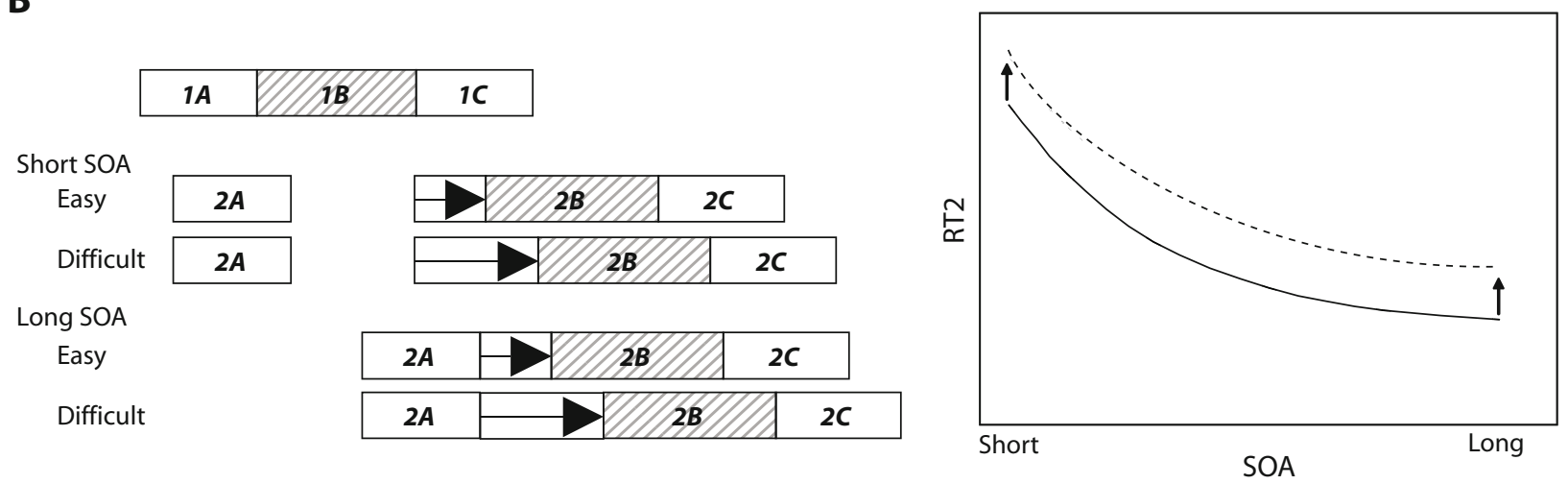

Figure 2. Two possible predictions of the central bottleneck model for manipulating at Task 2 stage duration. Panel A shows what would happen if the manipulated stage occurs prior to the bottleneck. At short stimulus onset asynchronies (SOAs), the effects of this manipulation should be greatly reduced—or even disappear-because they can be absorbed into cognitive slack. Panel B shows what would happen if the manipulated stage is subject to the bottleneck. The effects of this manipulation should be the same at all SOAs. $1 \mathrm{~A}, 1 \mathrm{~B}$, and $1 \mathrm{C}$ are the perceptual, central, and response initiation/execution stages of Task 1, respectively. $2 \mathrm{~A}, 2 \mathrm{~B}$, and $2 \mathrm{C}$ are the corresponding stages for Task 2. RT2, Task 2 response time. 
(Carrier \& Pashler, 1995), and certain difficult perceptual judgments (e.g., box-width judgment; Johnston \& McCann, 2006). That such a wide variety of mental processes are subject to the bottleneck supports the concept of a singlechannel, general-purpose resource - not unlike the CPU of a computer — which we call central attention for short.

\section{Emotion and Dual-Task Performance}

Emotional tasks (see Levenson, 1994) are tasks that require a person to process an emotional stimulus (e.g., interpreting the emotional expression of a human face) or that elicit an emotional response (e.g., crying after watching a sad movie). The present research focuses on the former - perception of an emotional expression - which we henceforth refer to as emotion perception. Regardless of whether emotional processes are fundamentally different from other cognitive processes (see Eder, Hommel, $\&$ De Houwer, 2007), we aim to determine whether these processes are automatic.

The usual everyday experience is that emotion perception is automatic in the sense of being effortless and beyond our control. We see a face and feel that we instantly perceive the expression, whether it be happy, sad, puzzled, or angry. This impression is supported by some empirical research (see Palermo \& Rhodes, 2007, for a review). But can emotion perception occur even when we are busy with another task? That is, does it require central attentional resources?

Surprisingly, previous PRP studies have not asked whether emotional processing requires central attention. Outside this literature, many previous studies have examined the role of "attention" in emotion perception, but have failed to make the important distinction between central attention and spatial attention (see Johnston, McCann, \& Remington, 1995; Pashler, 1991). Previous studies primarily manipulated spatial attention (focusing on a specific object or location in space), or some combination of spatial and central attention. These studies clearly indicated that emotion perception requires spatial attention (e.g., Pessoa, McKenna, Gutierrez, \& Ungerleider, 2002). However, these findings do not indicate whether emotion perception requires central attention.

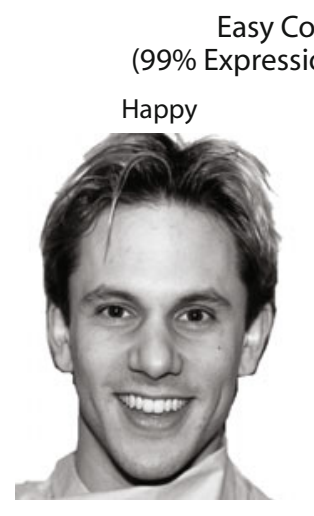

Easy Condition

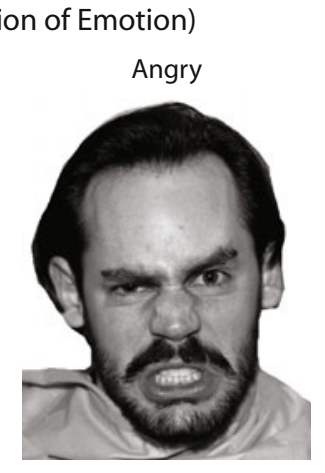

\section{EXPERIMENT 1}

Using locus-of-slack logic in a PRP paradigm, we examined whether people can perceive the emotional expression of a Task 2 stimulus (a photo of a face) while central resources are devoted to Task 1 . Task 1 was a speeded discrimination between a tone and a noise sound. Task 2 was a speeded discrimination of emotional facial expression: happy versus angry. One critical manipulation was the SOA between Task 1 and Task 2 . The other critical manipulation was the difficulty of the emotion discrimination. Half of the faces had an obviously happy or an obviously angry expression (easy condition), whereas the other half had a subtly happy or a subtly angry expression (difficult condition). Example stimuli are shown in Figure 3. As will be seen, the RT was $13 \mathrm{msec}$ longer, on average, in the difficult condition than in the easy condition.

We expect that Task 2 central operations (e.g., selecting a response) will wait for Task 1 central operations to finish, creating the usual central bottleneck (Figure 1). The critical question is whether emotional expressions can be processed in parallel with Task 1 central operations, which we addressed using locus-of-slack logic. If they can, then the effects of emotion-perception difficulty on Task 2 RT should be much smaller at short SOAs than at long SOAs (an underadditive interaction; Figure 2A). The reason is that at short SOAs, the extra time required for the difficult emotion perception should be absorbed into the cognitive slack created by the central bottleneck. But if emotion perception requires central resources, the effect of emotion-perception difficulty should be roughly the same at all SOAs (Figure 2B).

\section{Method}

Participants. Seventy-one University of New Mexico undergraduates participated for course credit. Eight participants were excluded because their Task 2 accuracy was less than $85 \%$. The remaining participants' mean age was 19.5 years.

Apparatus and Stimuli. Task 1 required a speeded discrimination between a pure tone $(22 \mathrm{kHz})$ and white noise. Task 2 required participants to determine whether a photograph of a human face expressed a happy or an angry emotion. From an average viewing distance of $60 \mathrm{~cm}$, the picture subtended a visual angle of $9^{\circ} \times 10.8^{\circ}$.

Figure 3. Examples of happy and angry faces used as stimuli in the easy and difficult conditions. The actual stimuli were in color. 
The faces were created by computer morphing, using photographs of happy, angry, and neutral expressions. ${ }^{1}$ The angry faces in the easy condition were a morphed mixture of $99 \%$ of an angry face and $1 \%$ of the corresponding neutral face. The angry faces in the difficult condition were a $50 \%$ mixture of the angry and neutral photographs. The happy faces were created in an analogous fashion. Figure 3 shows several examples. The end result is that the difficult versions expressed the emotion more subtly, yet looked natural (no participant remarked otherwise or commented on the use of computer morphing). There were eight models, each contributing to two stimuli (an easy and a hard version of an expression). In total, there were four face stimuli used in each of the four conditions (happyeasy, happy-difficult, angry-easy, and angry-difficult).

Design and Procedure. To begin each trial, a fixation point was placed in the center of the screen for $500 \mathrm{msec}$. Next, the Task 1 tone or noise was sounded, followed by the Task 2 face after a randomly selected SOA $(100,300,500$, or $900 \mathrm{msec})$.

For Task 1, participants pressed the " $\mathrm{z}$ " key for pure tones and the " $x$ " key for noise. For Task 2, half of the participants pressed the " $n$ " key for happy faces and the " $m$ " key for angry faces. For the other half, we reversed the assignment of the " $n$ " and " $m$ " keys to angry and happy, respectively. After each trial, feedback indicated whether the response was correct. If correct, the RT in milliseconds was also indicated. Participants were instructed to respond to each stimulus as quickly and as accurately as possible and to respond to Task 1 immediately, without waiting for Task 2.

Participants completed one practice block of 64 trials, followed by five experimental blocks of 128 trials each. The factors that were analyzed by an ANOVA were SOA $(100,300,500$, or $900 \mathrm{msec})$, Task 2 emotion (happy vs. angry), and Task 2 emotion-perception difficulty (difficult vs. easy). Conditions were selected at random with the restriction that each combination of SOA, emotion, and emotional difficulty should occur equally often within a session.

\section{Results and Discussion}

Individual trials were excluded if the RT to either task was below $200 \mathrm{msec}$ or above $3,500 \mathrm{msec}(0.71 \%$ of all trials). Error trials were eliminated from RT analyses. Table 1 shows the resulting mean RTs and error rates for Task 1 and Task 2. Figure 4 shows the critical relationship between the effects of SOA and emotion-perception difficulty on Task 2 RT.

No effects were significant for Task 1 RT, Task 1 error rate, or Task 2 error rate. However, an analysis of Task 2 RT revealed that participants responded $13 \mathrm{msec}$ slower in the difficult condition than in the easy condition
Table 1

Mean Response Times (RTs, in Milliseconds) and

Percentages of Error (PEs) for Task 1 and Task 2 As a

Function of Task 2 Difficulty and Stimulus Onset Asynchrony (in Milliseconds) in Experiment 1

\begin{tabular}{|c|c|c|c|c|c|c|c|c|}
\hline \multirow{3}{*}{$\begin{array}{c}\text { Task } 2 \\
\text { Difficulty }\end{array}$} & \multicolumn{8}{|c|}{ Stimulus Onset Asynchrony } \\
\hline & \multicolumn{2}{|c|}{100} & \multicolumn{2}{|c|}{300} & \multicolumn{2}{|c|}{500} & \multicolumn{2}{|c|}{900} \\
\hline & RT & $\overline{\mathrm{PE}}$ & RT & $\overline{P E}$ & RT & $\overline{P E}$ & RT & $\mathrm{PE}$ \\
\hline \multicolumn{9}{|c|}{ Task 1} \\
\hline Easy & 739 & 5 & 751 & 4 & 776 & 3 & 864 & 3 \\
\hline Difficult & 764 & 4 & 743 & 4 & 777 & 3 & 855 & 3 \\
\hline \multicolumn{9}{|c|}{ Task 2} \\
\hline Easy & 951 & 7 & 797 & 8 & 687 & 7 & 613 & 7 \\
\hline Difficult & 970 & 8 & 803 & 8 & 703 & 7 & 625 & 7 \\
\hline
\end{tabular}

$[F(1,62)=23.20, p<.0001]$. Mean RT increased as SOA decreased, indicating substantial dual-task interference $[F(3,186)=442.69, p<.0001]$; the overall PRP effect was 342 msec.

To examine whether emotions are processed without central resources, we looked for an underadditive interaction between SOA and Task 2 difficulty on Task 2 RT. Figure 4 shows no trend toward underadditivity $[F(3,186)=$ $1.18, p=.319]$; the effect of Task 2 difficulty was 18,6 , 16 , and $12 \mathrm{msec}$ at the 100-, 300-, 500-, and 900-msec SOAs, respectively.

This additive relationship is consistent with the null hypothesis of nonautomatic emotion perception. But to show that we can actually reject the alternative hypothesis of automatic emotion perception, a further analysis is required. Automatic emotion perception predicts the absence of Task 2 difficulty effects at the shortest SOA. The reason is that the very long period of cognitive slack created by the central bottleneck (note the large PRP effect of $364 \mathrm{msec}$ ) should be sufficient to fully absorb the small Task 2 difficulty effect (only $12 \mathrm{msec}$ at the longest SOA). Contrary to this prediction, the task difficulty effect at the shortest SOA was $18 \pm 14 \mathrm{msec}$ ( $95 \%$ confidence interval), which was significantly greater than $0[t(62)=2.26, p<.05]$. Thus, we can reject the hypothesis of fully automatic emotion perception.

\section{Experiment 1}

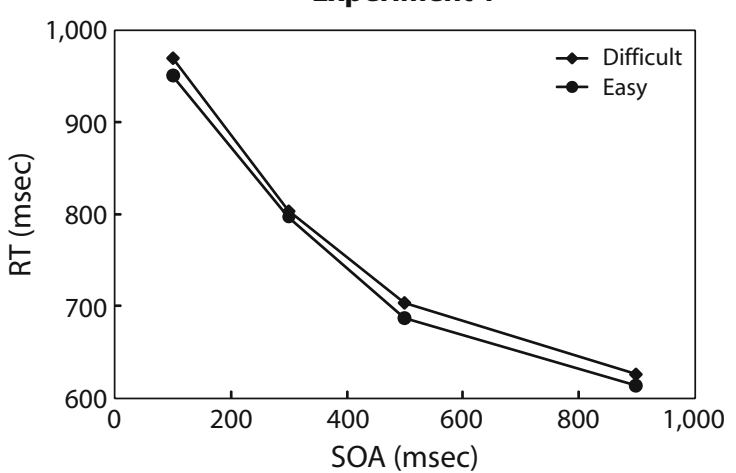

\section{Experiment 2}

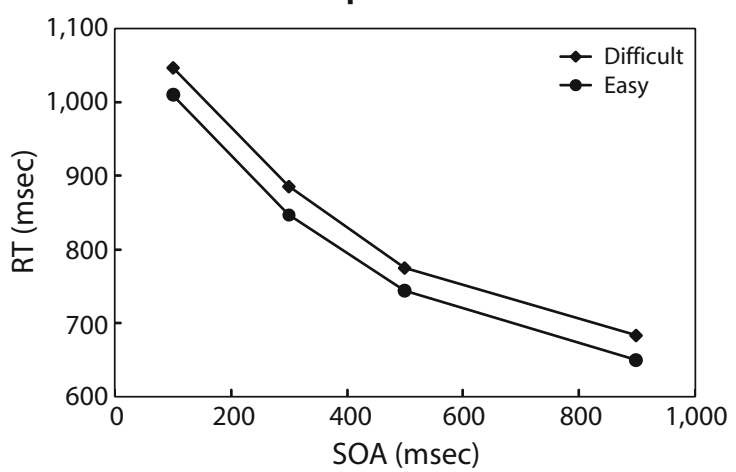

Figure 4. Mean response time (RT, in milliseconds) for Task 2 in Experiments 1 and 2 as a function of stimulus onset asynchrony (SOA, in milliseconds) and Task 2 difficulty. 


\section{EXPERIMENT 2}

In Experiment 1, the effect of our manipulation of emotion-perception difficulty was rather small—only $13 \mathrm{msec}$ - which perhaps made it difficult to detect any partial underadditivity. Therefore, Experiment 2 replicated Experiment 1 with a larger effect size.

A secondary concern is that, in Experiment 1, participants might have merely recognized the specific faces and pressed the corresponding button instead of truly processing the emotional content of the stimuli. To greatly reduce this concern, we increased the number of unique pictures from 16 to 40 . As will be seen, this change more than doubled the effect of the difficulty manipulation.

\section{Method \\ Participants. There were 70 participants; none had participated in Experiment 1. Three participants were excluded because their Task 2 accuracy was less than $85 \%$. The remaining participants' mean age was 20.1 years. \\ Apparatus, Stimuli, Procedure, and Design. Experiment 2 was similar to Experiment 1, except that the number of Task 2 face stimuli was increased. The stimuli included the 16 faces from Ex- periment 1 plus 24 new ones, for a total of 40 . The additional faces were drawn from the same source as the original 16. Participants completed two blocks of 320 trials, with a midblock break. Par- ticipants responded to Task 2 by pressing the " $n$ " and "b" keys. We counterbalanced the assignment of these keys to categories (happy vs. angry) across participants.}

\section{Results and Discussion}

Individual trials were excluded if the RT to either task was below $200 \mathrm{msec}$ or above $3,500 \mathrm{msec}(0.62 \%$ of all trials). The results are summarized in both Table 2 and Figure 4.

As in Experiment 1, no effects were significant for Task 1 RT, Task 1 error rate, or Task 2 error rate. The analysis of Task 2 RT showed that participants responded on average $35 \mathrm{msec}$ slower in the difficult condition than in the easy condition $[F(1,66)=57.21, p<.0001]$. There was also a main effect of SOA $[F(3,198)=492.63, p<$ $.0001]$; the overall PRP effect was $364 \mathrm{msec}$.

Again, we looked for an underadditive interaction between SOA and Task 2 difficulty. As in Experiment 1, there was no apparent trend toward underadditivity, and the interaction was nonsignificant $[F(3,198)=0.49, p<$

Table 2

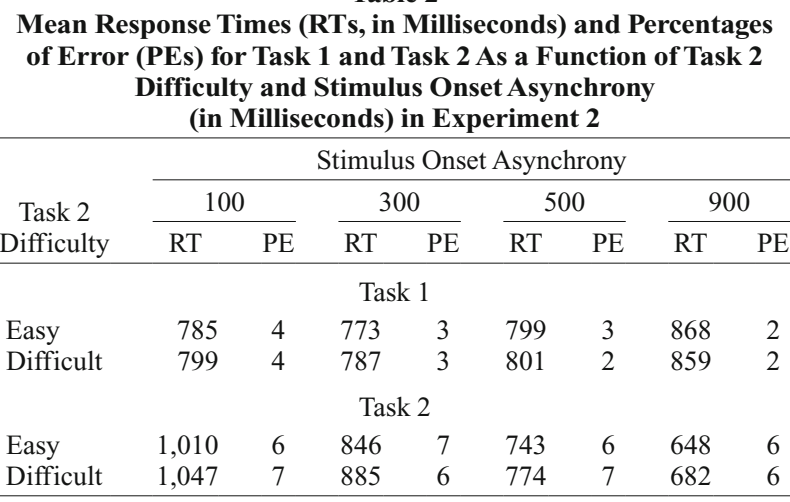

.691]. The effect of Task 2 difficulty was 37, 39, 31, and $34 \mathrm{msec}$ at the 100-, 300-, 500-, and 900-msec SOAs, respectively (see Figure 4).

The hypothesis of fully automatic emotion perception predicts that at short SOAs, the modest Task 2 difficulty effects should virtually disappear at short SOAs (note the large PRP effect of 364 msec, sufficient to absorb the 34msec Task 2 difficulty effect). Contrary to this prediction, the task difficulty effect at the shortest SOA was $37 \pm$ $17 \mathrm{msec}$ (95\% confidence interval), which was significantly greater than $0[t(66)=4.44, p<.001]$. This finding allows us to reject the hypothesis that emotion perception is fully automatic.

\section{GENERAL DISCUSSION}

Using locus-of-slack logic in a PRP paradigm, the present study addressed whether the perception of emotional expressions requires central resources. The critical difficulty manipulation was the degree of emotional expression (happy vs. angry) in the Task 2 face stimuli, creating an easy condition and a difficult condition (see Figure 3 for examples). Experiment 1 revealed an additive relationship between this manipulation of Task 2 emotion-perception difficulty and SOA. Experiment 2 replicated this finding using a stronger manipulation of emotion-perception difficulty. On the basis of locus-of-slack logic, this additivity suggests that the processing stage affected by our manipulation - which we call emotion perception-occurred only after the bottleneck delay (see Figure 2B).

These findings imply that either emotion perception or some prior stage cannot operate in parallel with attentiondemanding central operations on another task. Because no earlier stage seems like a plausible candidate, we conclude that it is emotion perception itself that cannot operate without central attention. In this sense, emotion perception is not fully automatic. This lack of automaticity is surprising, given the obvious biological importance of rapidly interpreting facial expressions to anticipate the intentions and likely actions of others.

\section{Relation to Previous Studies}

Previous studies have largely ignored the issue of whether emotion perception requires the central attentional resources engaged by response selection and other central processes. However, several previous studies have examined whether emotion perception can proceed even when people are not attending to that location in space (i.e., without spatial attention). Pessoa et al. (2002), for example, showed their participants emotional or neutral faces and asked them to identify whether the person was male or female or whether the orientation of bars in the periphery was the same or different. Pessoa et al. detected activation in the brain regions that are responsible for emotion processing (e.g., the amygdala) when the faces were spatially attended, but not when the bars were spatially attended. Thus, there is evidence that emotion perception requires spatial attention (see also Erthal et al., 2005; Okon-Singer, Tzelgov, \& Henik, 2007). 
In our study, Task 1 was auditory, presented through the left and right channels of the headphones. In principle, participants should have been able to maintain their spatial attention near the fixation point, where the Task 2 faces always appeared. Indeed, the results of previous studies have suggested that participants have little difficulty attending visual stimuli even while selecting a response to an auditory Task 1 stimulus (see, e.g., Johnston \& McCann, 2006; Pashler, 1984, 1991; Pashler \& Johnston, 1989). Thus, we believe that the problem is not that participants were looking in the wrong location (spatial attention), but rather that they were busy thinking about another task (central attention).

\section{Limitations of the Present Study}

The present study demonstrated the nonautomaticity of a particular emotional discrimination (happy vs. angry) using a particular type of stimuli (photographs of faces). It is, of course, conceivable that other forms of emotion perception would be more automatic. Note, however, that happy versus angry is a fundamental emotional discrimination. Also, our stimuli were rich (medium-resolution color photographs) and highly familiar (faces). One promising avenue for further study, however, might be the use of videos showing changes in emotional expression.

The additive data pattern, obtained in both experiments, was predicted by the hypothesis of completely nonautomatic emotion perception. Furthermore, the present findings argue against the hypothesis that emotion perception is fully automatic, because difficulty effects did not disappear at short SOAs. This finding does not imply, however, that absolutely no progress was made toward recognizing the emotions without central attention. First, although our experiments have far more than the usual numbers of participants, our data are nevertheless subject to sampling error. Pooling Experiments 1 and 2 together, we had sufficient power to detect even as little as a $50 \%$ reduction in difficulty effects at short SOAs, but we cannot rule out a reduction of, say, $25 \%$. Second, our data speak only to the automaticity of the particular stage of emotion processing influenced by our manipulation of the degree of emotional expression (see Figure 3 for examples). Perhaps some very early stages of emotional processing are automatic, even though later stages are not. Third, it is possible that facial expression was represented fully automatically somewhere in the brain (e.g., the amygdala). Further research using other measures (e.g., fMRI) is needed to evaluate this possibility. However, if any such representations are formed without central attention, they cannot easily be accessed by conscious cognition.

\section{Implications}

Many previous studies have suggested that the perception of emotional expressions in faces can be rapid, nonconscious, involuntary, and mandatory (for a review, see Palermo \& Rhodes, 2007). However, emotion perception does not meet all of the usual criteria for automaticity, because it appears to require limited attentional resources.
The present study indicates that emotion perception requires central attentional resources, and previous studies have indicated that it requires spatial attention (see, e.g., Pessoa et al., 2002).

A practical implication of the present findings is that people will be slow to perceive emotions - and slow to act appropriately on them - when they are busy with another task. For instance, when conversing at some social function, one might be slow to notice anger in an ignored companion. This conclusion runs counter to the common belief (among laypersons) that emotions are perceived automatically. Perhaps the same central bottleneck that delays emotion perception also impairs our ability to notice the delays.

\section{AUTHOR NOTE}

We thank Bruce Smith for providing the face stimuli used in the experiments. We are also grateful to Emily Gardner, Oksana Grochowski, Heather Macmillan, Jeremy Oat, Sean Rix, and Consuelo Salazar for their help collecting data. Correspondence concerning this article should be addressed to E. Ruthruff, Department of Psychology, University of New Mexico, Albuquerque, NM 87131 (e-mail: ruthruff@unm.edu).

\section{REFERENCES}

Carrier, L. M., \& Pashler, H. (1995). Attentional limits in memory retrieval. Journal of Experimental Psychology: Learning, Memory, \& Cognition, 21, 1339-1348.

Eder, A. B., Hommel, B., \& De Houwer, J. (2007). How distinctive is affective processing? On the implications of using cognitive paradigms to study affect and emotion. Cognition \& Emotion, 21, 11371154.

Erthal, F. S., De Oliviera, L., Mocaiber, I., Pereira, M. G., Machado-Pinheiro, W., Volchan, E., \& Pessoa, L. (2005). Loaddependent modulation of affective picture processing. Cognitive, Affective, \& Behavioral Neuroscience, 5, 388-395.

Johnston, J. C., \& McCann, R. S. (2006). On the locus of dual-task interference: Is there a bottleneck at the stimulus classification stage? Quarterly Journal of Experimental Psychology, 59, 694-719.

Johnston, J. C., McCann, R. S., \& Remington, R. W. (1995). Chronometric evidence for two types of attention. Psychological Science, 6, 365-369

JoliCEur, P. (1998). Modulation of the attentional blink by on-line response selection: Evidence from speeded and unspeeded Task decisions. Memory \& Cognition, 26, 1014-1032.

LEVENSON, R. (1994). Human emotion: A functional review. In P. Ekman \& R. J. Davidson (Eds.), The nature of emotion (pp. 123-126). New York: Oxford University Press.

Lien, M.-C., \& Proctor, R. W. (2002). Stimulus-response compatibility and psychological refractory period effects: Implications for response selection. Psychonomic Bulletin \& Review, 9, 212-238.

Lien, M.-C., Ruthruff, E., Cornett, L., Goodin, Z., \& Allen, P. A. (2008). On the nonautomaticity of visual word processing: Electrophysiological evidence that word identification requires central attention. Journal of Experimental Psychology: Human Perception \& Performance, 34, 751-773.

McCann, R. S., \& Johnston, J. C. (1992). Locus of single-channel bottleneck in dual-task interference. Journal of Experimental Psychology: Human Perception \& Performance, 18, 471-484.

McCann, R. S., Remington, R. W., \& Van Selst, M. (2000). A dualtask investigation of automaticity in visual word processing. Journal of Experimental Psychology: Human Perception \& Performance, 26, 1352-1370

Meyer, D., \& Kieras, D. (1997). A computational theory of executive cognitive processes and multiple-task performance: Part 2. Accounts of psychological refractory-period phenomena. Psychological Review, 104, 749-791.

OKon-Singer, H., Tzelgov, J., \& Henik, A. (2007). Distinguishing 
between automaticity and attention in the processing of emotionally significant stimuli. Emotion, 7, 147-157.

Palermo, R., \& Rhodes, G. (2007). Are you always on my mind? A review of how face perception and attention interact. Neuropsychologia, 45, 75-92.

PASHLER, H. (1984). Processing stages in overlapping tasks: Evidence for a central bottleneck. Journal of Experimental Psychology: Human Perception \& Performance, 10, 358-377.

PASHLER, H. (1991). Shifting visual attention and selecting motor responses: Distinct attentional mechanisms. Journal of Experimental Psychology: Human Perception \& Performance, 17, 1023-1040.

Pashler, H., \& Johnston, J. C. (1989). Chronometric evidence for central postponement in temporally overlapping tasks. Quarterly Journal of Experimental Psychology, 41A, 19-45.

Pessoa, L., McKenna, M., Gutierrez, E., \& Ungerleider, L. G. (2002). Neural processing of emotional faces requires attention. Proceedings of the National Academy of Sciences, 99, 11458-11463.

Ruthruff, E., Miller, J. O., \& Lachmann, T. (1995). Does mental rotation require central mechanisms? Journal of Experimental Psychology: Human Perception \& Performance, 21, 552-570.

SCHWEICKERT, R. (1978). A critical path generalization of the additive factor method: Analysis of a Stroop task. Journal of Mathematical Psychology, 18, 105-139.

Tombu, M., \& JoliceEur, P. (2003). A central capacity sharing model of dual-task performance. Journal of Experimental Psychology: Human Perception \& Performance, 29, 3-18.

\section{NOTE}

1. The morphed faces were developed by Bruce Smith, Department of Psychology, 1 University of New Mexico, MSC03 2220, Albuquerque, NM 87131-1161.

(Manuscript received May 2, 2008;

revision accepted for publication October 20, 2008.) 